\title{
Intensive versus conservative glycaemic control in patients undergoing coronary artery bypass graft surgery: a protocol for systematic review and meta- analysis of randomized controlled trials
}

\author{
Yi Liu \\ Shandong Qianfoshan Hospital \\ Meng Lv \\ Shandong Qianfoshan Hospital \\ Xia-xuan Sun \\ Shandong Qianfoshan Hospital \\ Wen-ya Du \\ Shandong Qianfoshan Hospital \\ Ting-ting Chen \\ Shandong Qianfoshan Hospital \\ Yuelan Wang ( $\nabla$ qymz8535@163.com ) \\ Shandong University
}

\section{Protocol}

Keywords: Glycaemic control, Coronary artery bypass graft, Systematic review, Meta-analysis, Protocol

Posted Date: November 23rd, 2021

DOI: https://doi.org/10.21203/rs.3.rs-1088323/v1

License: (a) (i) This work is licensed under a Creative Commons Attribution 4.0 International License.

Read Full License 


\section{Abstract \\ Background}

Coronary artery bypass graft (CABG) surgery has become the most common surgical procedure among all cardiac surgeries. Hyperglycaemia and hypoglycaemia occur frequently during this type of surgery and are associated with a variety of postoperative outcomes. Therefore, intraoperative glycaemic control is an important issue for patients undergoing CABG surgery. This systematic review and meta-analysis aim to evaluate the effects of different intraoperative glycaemic control strategies on postoperative outcomes.

\section{Methods}

We will perform a systematic review and meta-analysis of randomized controlled trials (RCTs) according to the recommendations of the Preferred Reporting Items for Systematic Reviews and Meta-Analyses (PRISMA) statement. Relevant studies will be searched in the Medline, Embase, Cochrane Library and Web of Science databases. Two independent reviewers will conduct study selection, data extraction, risk of bias assessment and quality of evidence assessment. The primary outcome will be postoperative mortality, and the secondary outcomes will include time on a ventilator in the intensive care unit (ICU), incidence of postoperative myocardial infarction (MI), incidence of postoperative atrial fibrillation (AF), rate of postoperative infection, incidence of acute kidney failure (AKF), length of stay (LOS) in the ICU, and LOS in the hospital. $\chi^{2}$ (Cochran Q) and $\mathrm{I}^{2}$ methods will be used to assess statistical heterogeneity. Sensitivity analysis will also be performed to explore the source of clinical heterogeneity, and subgroup analyses will be performed to detect possible heterogeneity of the results. Review Manager (RevMan) 5.4 will be used for data management and statistical analysis. The Cochrane risk-of-bias tool 2.0 and GRADEpro will be applied for risk of bias and quality assessment of the evidence.

\section{Discussion}

We assume that intensive glycaemic control in patients undergoing CABG will reduce postoperative mortality and complications, which seriously compromise a patient's quality of life and may even cause premature death. To address limitations in our study, corresponding methodologies will be adopted to optimize the results as much as possible. The results of our study are expected to provide strong evidence supporting the relationship between intensive intraoperative glycaemic control strategies and the reduction of postoperative complications in patients undergoing CABG surgery.

\section{Systematic review registration}

PROSPERO CRD42021240841. 


\section{Background}

Hyperglycaemia in hospitalized patients (blood glucose more than $140 \mathrm{mg} / \mathrm{dL}$ or $7.8 \mathrm{mmol} / \mathrm{L}$ ) is commonly encountered during the perioperative period and is estimated to occur in $20-40 \%$ of noncardiac patients, in $35 \%$ of vascular patients, and in $80 \%$ of cardiac surgery patients [1]. Coronary artery bypass graft (CABG) surgery has become the most common cardiac surgery, and almost 400,000 patients undergo $C A B G$ surgery annually [2]. Previous data have shown that hyperglycaemia during cardiac surgery is associated with hospital complications, including mortality, renal failure, wound infections and duration of mechanical ventilation, especially if it is not well controlled [3-10]. In addition, hyperglycaemia is thought to increase perioperative morbidity and mortality [11].

To address intraoperative hyperglycaemia, an intensive glycaemic control strategy is often adopted, but it easily causes the occurrence of hypoglycaemia. It has been suggested that there may be a significant reduction in early mortality among patients undergoing CABG surgery with intensive glycaemic control [12-15]. However, other evidence of severe hypoglycaemia resulting from intensive intraoperative glycaemic control brings the safety and effectiveness of this management strategy into question $[8,11$, 16]. Hypoglycaemia has also been considered an independent risk factor for undesired clinical outcomes and hospital mortality [17-19]. Several studies have confirmed that the incidence of hypoglycaemia is associated with the overall risk of hospital mortality and an increased risk of cardiovascular events in critically ill patients [10, 20-22].

Protocols for intraoperative glycaemic management during CABG surgery have been established and implemented to improve poor postoperative outcomes [23, 24]. However, the optimal strategy for intraoperative glycaemic control during CABG surgery remains controversial.

Given the confusing evidence of the association between intraoperative glycaemic control strategies and postoperative complications, we will employ this systematic review and meta-analysis to evaluate it. Eligible randomized controlled trials (RCTs) will be strictly selected in our study. We will develop inclusion and exclusion criteria, which will include study design, population, intervention, comparator and outcome, to ensure the quality and effectiveness of our systematic review.

\section{Methods}

\section{Protocol design and registration}

Our study has been registered within the PROSPERO International Prospective Register of Systematic Reviews. The registration number is CRD42021240841. The protocol is being reported according to the Preferred Reporting Items for Systematic Reviews and Meta-Analysis Protocols (PRISMA-P) 2015 checklist [25] (see checklist in Additional file 1). We will conduct our systematic review and meta-analysis following the Preferred Reporting Items for Systematic Reviews and Meta-analyses (PRISMA) guidelines [26]. 


\section{The primary and second outcomes}

The primary outcome will be postoperative mortality. The secondary outcomes will include time on a ventilator in the intensive care unit (ICU), incidence of postoperative myocardial infarction (MI), incidence of postoperative atrial fibrillation (AF), incidence of acute renal failure (AKF), rate of postoperative infection, and length of stay (LOS) in the ICU, and LOS in the hospital.

In our systematic review and meta-analysis, the outcomes will be defined as follows:

1) Postoperative mortality is defined as mortality within 30 days of the surgical procedure or during hospitalization due to causes related to the surgical procedure;

2) Time on a ventilator in the ICU is defined as the time from admission to the ICU to the time of extubation;

3) Incidence of postoperative MI and AF are defined as all reported occurrences of MI or AF after surgery, regardless of pervious status;

4) The incidence of AKF is defined as renal impairment needing postoperative dialysis or as twofold increase in creatinine over its baseline level;

5) Rate of postoperative infection refers to any reported infection after surgery, such as pneumonia and wound infection;

6) The length of stay (LOS) in the ICU is defined as the time from ICU admission to transfer out of the ICU. Before transfer, all patients were extubated and had stable vital signs and without any inotropic support;

7) LOS in the hospital was defined as the time from the day of surgery to the day of discharge.

If the above outcomes are reported at multiple time points, we will use the longest time frame reported.

\section{Eligibility criteria}

We will use the following eligibility criteria to select relevant studies:

1) Study design: Randomized controlled trials (RCTs) will be strictly screened. Crossover studies and quasi-randomized controlled trials will be excluded.

2) Population: Studies including patients undergoing CABG surgery who have accepted intraoperative glycaemic control will be included. Studies including patients with incomplete information will be excluded.

3) Intervention and comparator: Studies with at least two glycaemic control groups will be included. In the systematic review, the group with the lower blood glucose target will be defined as the 'intensive group', 
and the group with the higher blood glucose target will be defined as the 'conservative group'. Studies that did not measure blood glucose or did not have at least two groups will be excluded.

4) Outcome: Studies that evaluate any of our primary or second outcomes as endpoints will be included.

5) Language: Studies reported in all languages will be included. If necessary, scientifically trained reviewers will be consulted.

\section{Search strategy}

Two independent reviewers (YL and XXS) will perform comprehensive search strategies in the Medline, Embase, Cochrane Library and Web of Science databases. Records from inception to August 9, 2021, will be included. The search strategy will combine relevant Medical Subject Headings (MeSH) and keywords with synonyms and names of generic and brand names typically used in CABG surgery and glycaemic control. We will also check the reference lists of the included RCTs and relevant reviews. In addition, a grey literature search will be performed on trial registry sites (such as the WHO International Clinical Trial Registry Platform and NIH resources) and regulatory agency databases for unpublished studies. A manual search of citations and meeting reports will also be conducted. Duplicate publications will be excluded. If the same participants were used in multiple studies, we will include the study with the longest follow-up. The details of the search strategies of the four databases and the two trial registries are shown in Additional file 2.

\section{Study selection}

Endnote X9 will be applied to manage relevant articles and remove duplicates. To assess the eligibility of the potentially included studies, two independent reviewers will perform the study selection according to the predetermined inclusion and exclusion criteria. Then, they will check all titles/abstracts and the full text of each article independently and in duplicate. All disagreements will be resolved by discussion or by consulting a third reviewer (ML). During this period, all reasons for exclusion will be concretely recorded. If necessary, the reviewers will consult the methodological experts to reach consensus. A PRISMA 2020 flowchart will be adopted to record the process of study selection. It can be found in Additional file 3 .

\section{Data items}

The data will be extracted from the eligible studies. A predetermined data collection form will be used to collect the study data and will be pilot tested on a few randomly elected included studies. The author of a study without insufficient information will be contacted for further information. Two independent investigators will perform data extraction. In case of a disagreement, a third reviewer will be consulted. Arm-level data (e.g., number of events) will be extracted whenever possible. The predetermined data extraction form is be presented in Additional file 4.

\section{Risk of bias assessment}

According to the Cochrane Collaboration's risk-of-bias tool 2.0 for the quality randomized controlled trials [27], two investigators will independently determine the risk of bias on the included studies. The studies 
will be evaluated in three categories: low risk of bias, high risk of bias, and some concerns. Bias in the following domains will be assessed: bias arising from the randomization process, bias due to deviations from intended interventions, bias due to missing outcome data, bias in measurement of the outcome, and bias in selection of the reported result. In addition, we will reach an overall risk-of-bias judgement for each study based on the above assessments. RevMan 5.4 will be employed to graph and evaluate other biases [28].

\section{Measures of treatment effects}

The outcomes of the included studies will initially be grouped for analysis into either the primary outcome group or the secondary outcomes group. The primary outcome will be postoperative mortality. The secondary outcomes will be further categorized into time on a ventilator in the ICU, incidence of postoperative $\mathrm{MI}$, incidence of postoperative AF, rate of infection, incidence of AKF, LOS in the ICU, and LOS in the hospital. The level of blood glucose will be regarded as a binary variable because all eligible studies report their outcomes in this form. Each postoperative outcome will be estimated for inclusion in the intensive glycaemic control group or in the conservative glycaemic control group.

\section{Assessment of heterogeneity}

$\chi^{2}$ (Cochran Q) and $I^{2}$ methods will be used to assess statistical heterogeneity among the eligible studies. In accord with The Cochrane Handbook for Systematic Reviews of Interventions, $I^{2}$ statistics will be used to categorize the heterogeneity into nonimportant $(<30 \%)$, moderate $(30 \%-60 \%)$ and substantial $(>60 \%)$.

\section{Data synthesis}

The risk ratio (RR) will be used for both fixed- and random-effects models (weighting by inverse of variance). The primary outcome and secondary outcomes will be assessed using forest plots and presented as RRs. Sensitivity analyses will be performed to confirm the results of the meta-analyses by excluding each of the unqualified studies. A funnel plot for meta-analyses will be used to assess publication bias. If required, the Egger method will be used to quantify the publication bias. Statistical analyses will be performed using RevMan5.4 software (The Nordic Cochrane Centre, Copenhagen, Denmark). For continuous outcomes, weighted mean differences or standardized mean differences will be used according to different measurement scales. In our studies, the confidence interval (Cl) will be established at $95 \%$, and a $P$ value $<.05$ will be considered statistically significant.

\section{Subgroup analysis}

Subgroup analysis will be performed according to the primary and secondary outcomes. Subgroup analyses will be performed to detect possible heterogeneity of the results. We will investigate the effects in five subgroup analyses:

1) History of diabetes versus not accompanied by diabetes;

2) History of hypertension versus not accompanied by hypertension; 
3) History of smoking versus not accompanied by smoking;

4) Different glycaemic control strategies: insulin versus glucose-insulin-potassium (GIK);

5) Off-pump CABG versus on-pump CABG.

\section{Sensitivity analysis}

Sensitivity analysis will also be performed to explore the source of clinical heterogeneity. To confirm the robustness of our findings, a sensitivity analysis will be conducted based on the different levels of bias of the included studies. To evaluate the internal validity of studies or treatment adequacy, we will subsequently remove studies with 'high risk' of bias, studies with 'some concerns' of bias, and studies with 'low risk' of bias. In addition, both a random effect model and a fixed effect model will be used for the synthesis of primary and secondary outcomes.

\section{Meta-bias}

To determine whether reporting bias is present, we will explore whether the protocols of the studies included in this systematic review were published before the recruitment of patients was initiated. The reporting bias will be further determined by funnel plots if there are more than 10 studies in this systematic review.

\section{Confidence in cumulative estimate}

To appraise the quality of the treatment effect estimate, we will use the Grading of Recommendations Assessment, Development and Evaluation (GRADE) approach. We will rate the quality of evidence using the five GRADE categories: study limitations, consistency of effect, imprecision, indirectness and publication bias. We will then rate the quality of evidence of each effect estimate: high level, moderate level, low level and very low level [29]. GRADEpro software will be applied to perform quality assessment [30].

\section{Discussion}

Existing studies have different opinions about the association between different glycaemic control strategies and postoperative complications in patients undergoing CABG surgery. Previous data have shown that hyperglycaemia during cardiac surgery is associated with hospital complications and mortality $[4,6,7]$. However, hypoglycaemia resulting from intensive glycaemic control is also considered an independent risk factor for undesired perioperative outcomes and hospital mortality [17-19, 21]. To gain further insight into the effect of different glycaemic control strategies on the perioperative outcomes of patients undergoing CABG surgery, we will conduct a strict systematic review and meta-analysis.

In this study, we will perform a comprehensive database search to select all eligible RCTs according to predetermined criteria. Risk of bias will be applied to evaluate the included literature, while assessment of heterogeneity, subgroup analysis and sensitivity analysis will be used to explore heterogeneity and its 
sources in the eligible studies. Our systematic review and meta-analysis will employ data synthesis to further explore the effect of glycaemic management on postoperative outcomes. Thus, the results of our study will provide reliable evidence for the association between different glycaemic control strategies and postoperative complications. Moreover, our conclusions from this systematic meta-analysis will determine the optimal intraoperative glycaemic management strategy for patients undergoing CABG surgery.

There are several limitations in our study. First, some perioperative complications of CABG were probably only reported in one or two studies, which limits the meta-analysis for these complications. The reasons may be the low rate of complications or something else. Second, the level of blood glucose will be treated as a binary variable in different strategies of glycaemic control, but blood glucose is, in fact, a continuous variable. Therefore, to respect the original studies that reported complications in two groups classified by the blood glucose level, we will conduct our research using the same classification method. Third, a few of the eligible studies included in our systematic review may be of low quality. We will perform sensitivity analysis for this situation.

\section{Abbreviations}

CABG

coronary artery bypass graft

ICU

intensive care unit

MI

myocardial infarction

$\mathrm{AF}$

atrial fibrillation

AKF

acute kidney failure

LOS

length of stay

RCTs

randomized controlled trials

RR

risk ratio

$\mathrm{Cl}$

confidence intervals

$\mathrm{MeSH}$

Medical Subject Headings.

\section{Declarations}


Acknowledgements

The authors would like to thank the First Affiliated Hospital of Shandong First Medical University for their assistance and support in conducting this systematic review.

\section{Authors' contributions}

$Y L$ and $M L$ initiated the study. YL, ML, XXS, and WYD participated in the study design. YL, XXS and WYD contributed to the development of inclusion criteria, data extraction and search strategies for CABG surgery and glycaemic control. YL wrote the draft of the protocol, and ML provided valuable suggestions. ML and TTC provided important advice on statistics and data analysis. All authors responded to reviewers' comments together. The final version of the protocol was approved by all authors.

\section{Funding}

This work was supported by the Natural Science Foundation of Shandong Province (ZR2016HL02).

\section{Availability of data and materials}

Not applicable.

\section{Declarations}

\section{Ethics approval and consent to participate}

Not applicable.

\section{Consent for publication}

Not applicable.

\section{Competing interests}

The authors declare that they have no competing interests.

\section{Author details}

${ }^{3}$ Department of Anaesthesiology, the First Affiliated Hospital of Shandong First Medical University \& Shandong Provincial Qianfoshan Hospital, Jinan, Shandong, 250014, China.

\section{References}

1. Duggan E, Chen Y. Glycemic Management in the Operating Room: Screening, Monitoring, Oral Hypoglycemics, and Insulin Therapy. Current diabetes reports. 2019;19(11):134. 
2. Alexander JH, Smith PK. Coronary-Artery Bypass Grafting. The New England journal of medicine. 2016;374(20):1954-64.

3. Furnary AP, Wu Y. Clinical effects of hyperglycemia in the cardiac surgery population: the Portland Diabetic Project. Endocrine practice : official journal of the American College of Endocrinology and the American Association of Clinical Endocrinologists. 2006;12 Suppl 3:22-6.

4. Furnary AP, Gao G, Grunkemeier GL, Wu Y, Zerr KJ, Bookin SO, et al. Continuous insulin infusion reduces mortality in patients with diabetes undergoing coronary artery bypass grafting. The Journal of thoracic and cardiovascular surgery. 2003;125(5):1007-21.

5. Kitabchi AE, Freire AX, Umpierrez GE. Evidence for strict inpatient blood glucose control: time to revise glycemic goals in hospitalized patients. Metabolism: clinical and experimental. 2008;57(1):116-20.

6. Hassan E. Hyperglycemia management in the hospital setting. American journal of health-system pharmacy : AJHP : official journal of the American Society of Health-System Pharmacists. 2007;64(10 Suppl 6):S9-s14.

7. van den Berghe $G$, Wouters $P$, Weekers F, Verwaest $C$, Bruyninckx F, Schetz $M$, et al. Intensive insulin therapy in critically ill patients. The New England journal of medicine. 2001;345(19):1359-67.

8. Finfer S, Chittock DR, Su SY, Blair D, Foster D, Dhingra V, et al. Intensive versus conventional glucose control in critically ill patients. The New England journal of medicine. 2009;360(13):1283-97.

9. Lazar HL, McDonnell M, Chipkin SR, Furnary AP, Engelman RM, Sadhu AR, et al. The Society of Thoracic Surgeons practice guideline series: Blood glucose management during adult cardiac surgery. The Annals of thoracic surgery. 2009;87(2):663-9.

10. Lazar HL, Chipkin SR, Fitzgerald CA, Bao Y, Cabral H, Apstein CS. Tight glycemic control in diabetic coronary artery bypass graft patients improves perioperative outcomes and decreases recurrent ischemic events. Circulation. 2004;109(12):1497-502.

11. Desai SP, Henry LL, Holmes SD, Hunt SL, Martin CT, Hebsur S, et al. Strict versus liberal target range for perioperative glucose in patients undergoing coronary artery bypass grafting: a prospective randomized controlled trial. The Journal of thoracic and cardiovascular surgery. 2012;143(2):318-25.

12. Haga KK, McClymont KL, Clarke S, Grounds RS, Ng KY, Glyde DW, et al. The effect of tight glycaemic control, during and after cardiac surgery, on patient mortality and morbidity: A systematic review and meta-analysis. Journal of cardiothoracic surgery. 2011;6:3.

13. Gerstein HC, Miller ME, Byington RP, Goff DC, Jr., Bigger JT, Buse JB, et al. Effects of intensive glucose lowering in type 2 diabetes. The New England journal of medicine. 2008;358(24):2545-59. 
14. Mechanick JI, Handelsman Y, Bloomgarden ZT. Hypoglycemia in the intensive care unit. Current opinion in clinical nutrition and metabolic care. 2007;10(2):193-6.

15. Ouattara A, Lecomte $P$, Le Manach $Y$, Landi $M$, Jacqueminet $S$, Platonov I, et al. Poor intraoperative blood glucose control is associated with a worsened hospital outcome after cardiac surgery in diabetic patients. Anesthesiology. 2005;103(4):687-94.

16. Gandhi GY, Nuttall GA, Abel MD, Mullany CJ, Schaff HV, O'Brien PC, et al. Intensive intraoperative insulin therapy versus conventional glucose management during cardiac surgery: a randomized trial. Annals of internal medicine. 2007;146(4):233-43.

17. Turchin A, Matheny ME, Shubina M, Scanlon JV, Greenwood B, Pendergrass ML. Hypoglycemia and clinical outcomes in patients with diabetes hospitalized in the general ward. Diabetes care. 2009;32(7):1153-7.

18. Kagansky N, Levy S, Rimon E, Cojocaru L, Fridman A, Ozer Z, et al. Hypoglycemia as a predictor of mortality in hospitalized elderly patients. Archives of internal medicine. 2003;163(15):1825-9.

19. Stagnaro-Green A, Barton MK, Linekin PL, Corkery E, deBeer K, Roman SH. Mortality in hospitalized patients with hypoglycemia and severe hyperglycemia. The Mount Sinai journal of medicine, New York. 1995;62(6):422-6.

20. Egi M, Bellomo R, Stachowski E, French CJ, Hart GK, Taori G, et al. Hypoglycemia and outcome in critically ill patients. Mayo Clinic proceedings. 2010;85(3):217-24.

21. Krinsley JS, Grover A. Severe hypoglycemia in critically ill patients: risk factors and outcomes. Critical care medicine. 2007;35(10):2262-7.

22. Preiser JC, Brunkhorst F. Tight glucose control and hypoglycemia. Critical care medicine. 2008;36(4):1391; author reply -2.

23. Lazar HL, McDonnell MM, Chipkin S, Fitzgerald C, Bliss C, Cabral H. Effects of aggressive versus moderate glycemic control on clinical outcomes in diabetic coronary artery bypass graft patients. Annals of surgery. 2011;254(3):458-63; discussion 63-4.

24. Zerr KJ, Furnary AP, Grunkemeier GL, Bookin S, Kanhere V, Starr A. Glucose control lowers the risk of wound infection in diabetics after open heart operations. The Annals of thoracic surgery. 1997;63(2):35661.

25. Moher D, Shamseer L, Clarke M, Ghersi D, Liberati A, Petticrew M, et al. Preferred reporting items for systematic review and meta-analysis protocols (PRISMA-P) 2015 statement. Systematic reviews. 2015;4(1):1 . 
26. Page MJ, McKenzie JE, Bossuyt PM, Boutron I, Hoffmann TC, Mulrow CD, et al. The PRISMA 2020 statement: an updated guideline for reporting systematic reviews. Systematic reviews. 2021;10(1):89.

27. Sterne JAC, Savović J, Page MJ, Elbers RG, Blencowe NS, Boutron I, et al. RoB 2: a revised tool for assessing risk of bias in randomised trials. BMJ (Clinical research ed). 2019;366:14898.

28. Review Manager Web (RevMan Web): The Cochrane Collaboration, Copenhagen, Denmark; [updated September 2020. Available from: https://revman.cochrane.org.

29. Puhan MA, Schünemann HJ, Murad MH, Li T, Brignardello-Petersen R, Singh JA, et al. A GRADE Working Group approach for rating the quality of treatment effect estimates from network meta-analysis. BMJ (Clinical research ed). 2014;349:g5630.

30. GRADEpro GDT: GRADEpro Guideline Development Tool: McMaster University, Hamilton, Canada; [updated 2021. Developed by Evidence Prime, Inc.]. Available from: https://gradepro.org.

\section{Supplementary Files}

This is a list of supplementary files associated with this preprint. Click to download.

- Additionalfile1.PRISMAP2015Checklist.docx

- Additionalfile2.DraftofSearchStrategies.docx

- Additionalfile3.PRISMA2020FlowDiagram.docx

- Additionalfile4.DraftofDataExtractionTemplate.docx 